\title{
Instabilidade do Carpo
}

\section{Carpal Instability}

\section{Leonardo Massamaro Sugawara ${ }^{(1)}$, Matiko Yanaguizawa ${ }^{(1)}$, Marcos Hiroyuki Ikawa $^{(1)}$, Rogério Diniz Takahashi ${ }^{(1)}$, Jamil Natour ${ }^{(2)}$, Artur da Rocha Corrêa Fernandes ${ }^{(1)}$}

\section{INTRODUÇÃO}

Lesões agudas e repetitivas no punho estão associadas à instabilidade de punho. A instabilidade pode ainda estar relacionada a outras comorbidades como a osteonecrose, doenças inflamatórias crônicas (artrite reumatóide), artropatias microcristalinas (doença por depósito de pirofosfato de cálcio), doenças neurológicas (seringohidromielia), doenças neoplásicas, além de malformações congênitas específicas como a deformidade de Madelung. Fratura do rádio e escafóide também pode estar relacionada à instabilidade $^{(1)}$.

O punho é uma estrutura complexa, composta de ossos, ligamentos e tendões que juntos e de forma harmoniosa permite movimentos nos planos coronal e sagital ${ }^{(2)}$. Em virtude de seu posicionamento e de seu arco de movimento, o punho é suscetível a constantes forças axiais e vetores de deformação.

A estabilidade carpal é definida como a habilidade do punho em manter o balanço estático e dinâmico entre as articulações sob as cargas fisiológicas e movimentos ${ }^{(1)}$. Já a instabilidade é o distúrbio desse balanço, relacionado às lesões ósseas $\mathrm{e} / \mathrm{ou}$ ligamentares, que resulta em incapacidade de manter as relações anatômicas articulares, ocasionando déficit da performance biomecânica, dor e colapso $\operatorname{carpal}^{(3)}$.

\section{ASPECTOS CLÍNICOS}

O quadro clínico é bastante variável, mas usualmente há relato de dor, perda de força ou estalido ao utilizar a mão. Edema pode estar relacionado e ser erroneamente interpretado como um cisto sinovial dorsal. A limitação de movimento ocorre comumente nas fases tardias. O diagnóstico precoce é essencial para melhor prognóstico, porém a maioria dos pacientes não procura atendimento inicial em razão de trauma parecer corriqueiro $^{(4)}$.

\section{ASPECTOS FUNCIONAIS DA ANATOMIA ÓSSEA E LIGAMENTAR}

A porção óssea do punho é composta por duas fileiras de ossos, a fileira proximal e a distal.

A fileira proximal, também chamada de segmento intercalado, contém os ossos escafóide, semilunar e piramidal, e representa o côndilo carpal, articulando-se com o antebraço por meio do rádio e da fibrocartilagem triangular.

A fileira distal contém os ossos trapézio, trapezóide, capitato e hamato, formando uma unidade com movimentação limitada.

O complexo ligamentar do punho pode ser classificado em extrínseco, ligamentos que unem o antebraço e o carpo, e intrínseco, ligamentos com origem e inserção no $\operatorname{carpo}^{(5)}$.

\section{LIGAMENTOS EXTRÍNSECOS}

Há três principais ligamentos radiocarpais palmares, $O$ ligamento radioescafóide, radiolunopiramidal e o radiosemilunar, e apenas um ligamento dorsal importante, o ligamento radiocárpico dorsal. Esses ligamentos fixam-se na fileira proximal e estabilizam a articulação radiocárpica ${ }^{(5)}$.

\section{LIGAMENTOS INTRÍNSECOS}

Os ligamentos escafosemilunar (LES) e semilunarpiramidal (LSP) são os ligamentos mais importantes, responsáveis pela estabilização da fileira proximal. Esses ligamentos fixam a margem proximal do escafóide e o semilunar e o semilunar proximal ao piramidal, respectivamente ${ }^{(5)}$.

O punho sustenta não só as forças externas, mas também as forças resultantes de contração muscular.

Declaramos a inexistência de conflitos de interesse.

1. Departamento de Diagnóstico por Imagem da Universidade Federal de São Paulo (Unifesp).

2. Disciplina de Reumatologia da Unifesp.

Endereço para correspondência: Artur da Rocha Corrêa Fernandes, DDI/Unifesp, Rua Botucatu, 740, CEP 04023-900, São Paulo, SP, e-mail: artur_personal@yahoo.com 
A fileira proximal é também denominada de segmento intercalado, por não possuir inserção tendínea, e sua movimentação é determinada pela força muscular exercida no rádio e na fileira distal ${ }^{(1,4)}$.

A fileira distal é considerada como uma unidade funcional em razão de sua conexão ligamentar. Como nenhum tendão é inserido na fileira proximal, todos os eixos de rotação são localizados na fileira distal, onde o movimento inicia-se.

$\mathrm{Na}$ fileira proximal, sob força axial, o escafóide tem a tendência à flexão, e o semilunar e o piramidal tendem à extensão $\mathrm{O}^{(1)}$.

\section{AVALIAÇÃO POR IMAGEM}

\section{RADIOGRAFIA CONVENCIONAL}

Para uma boa interpretação, é necessário que as radiografias sejam realizadas com técnica correta com o punho em posição neutra nas incidências de frente (posteroanterior) e perfil.

Na radiografia de frente, três arcos harmônicos e contínuos podem ser traçados (arcos de Gilula) ao longo dos ossos do carpo (Figura 1). Quando um desarranjo é evidente, esses arcos perdem a configuração ou podem ficar descontínuos ${ }^{(6)}$.

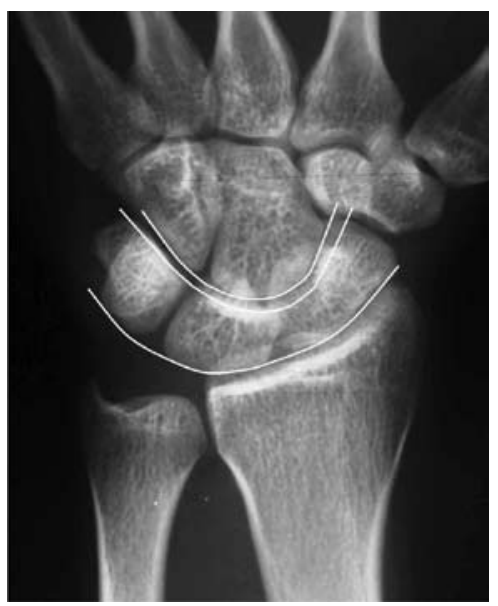

Figura 1 - Radiografia posteroanterior. Arcos de Gilula em paciente normal. Três arcos paralelos são observados, delineando as superfícies articulares radiocarpal e intercarpal proximal.

Nas radiografias em perfil, é possível a avaliação do alinhamento dos ossos do carpo, traçando-se os ângulos semilunarcapitato (normal até $30^{\circ}$ ) e escafosemilunar (normal de $30^{\circ}$ a $60^{\circ}$ ). Em um punho normal, os eixos longos do rádio, semilunar, capitato e o terceiro metacarpal são colineares $^{(1)}$ (Figuras 2a e 2b).

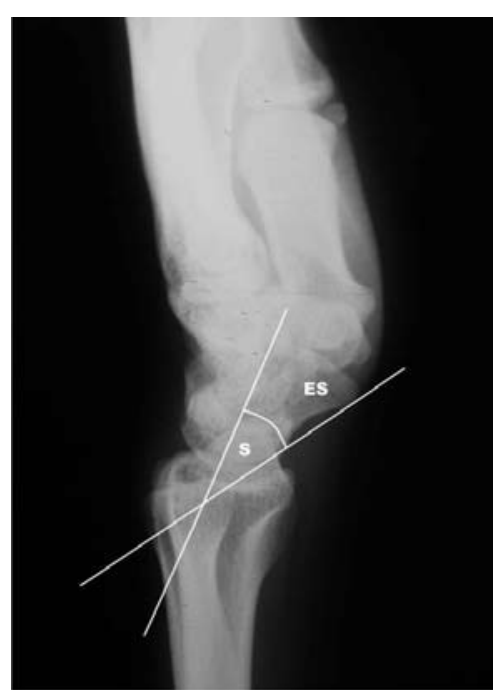

Figura 2a - Radiografia em perfil. 0 ângulo escafosemilunar normal $\left(30^{\circ}\right.$ a $\left.60^{\circ}\right)$ foi determinado traçando-se duas linhas ao longo do maior eixo do escafóide e do semilunar.

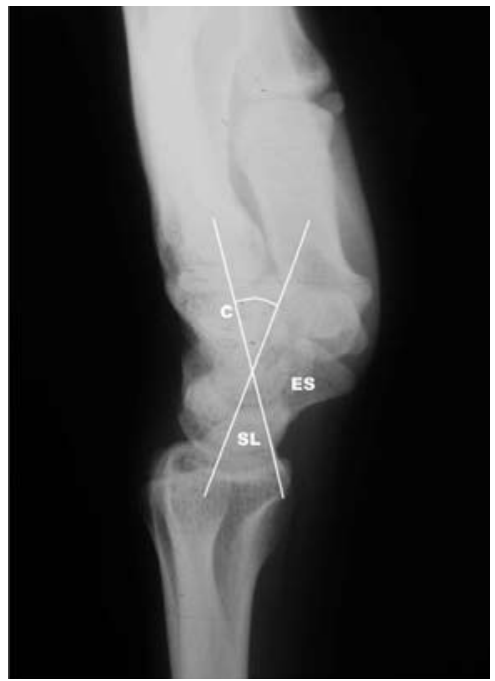

Figura $2 \mathrm{~b}$ - Mesmo paciente, porém traçando-se o ângulo semilunarcapitato normal por meio de duas linhas ao longo dos eixos maiores do semilunar e capitato. Normal de até $30^{\circ}$. Escafóide (ES), semilunar (SL) e capitato (C).

\section{RADIOGRAFIAS DINÂMICAS}

Na presença de uma instabilidade dinâmica, as radiografias convencionais podem estar normais. Desse modo, as radiografias devem ser realizadas sob carga máxima. Podem ser utilizadas três outras incidências sob estresse: radiografias 
posteroanteriores (PA) de frente com desvio ulnar e radial do punho e com o punho cerrado. O desvio ulnar máximo sobrecarrega o LES e exacerba lesões preexistentes. A incidência com punho fechado aumenta a carga no capitato, forçando o intervalo escafosemilunar. Qualquer alteração deve ser comparada com o punho contralateral ${ }^{(4)}$.

Outro método é a utilização da cineradioscopia, que consiste na realização dos exames com estresse em tempo real com a utilização de fluoroscopia. Neste exame dinâmico, é possível simular o movimento que gera a instabilidade. A perda do sincronismo dos movimentos das articulações pode ser demonstrada ${ }^{(1)}$.

\section{ARTROGRAFIA}

A artrografia do punho permite a detecção de defeitos ligamentares e da fibrocartilagem triangular por meio da injeção de contraste nos três compartimentos do punho. A artrografia possui uma sensibilidade de cerca de $60 \%$ para lesão do LES e tem sido substituída pela artroscopia ${ }^{(4)}$.

\section{RESSONÂNCIA MAGNÉTICA}

A ressonância magnética $(\mathrm{RM})$, em virtude de sua resolução anatômica, permite a visualização direta dos ligamentos e a avaliação da cortical e da medular óssea. Em razão da complexidade ligamentar, é um exame de difícil interpretação. Relatos na literatura sugerem uma sensibilidade de $63 \%$ e especificidade de $86 \%$ para a detecção de lesão no LES (Figura 3$)^{(4)}$. O uso de contraste não demonstrou melhora no diagnóstico ${ }^{(1)}$.

É possível utilizar contraste diretamente na articulação, semelhante à artrografia, para a realização da artro-RM, melhorando a acurácia para lesões ligamentares ${ }^{(1)}$.

\section{TOMOGRAFIA COMPUTADORIZADA}

Com o advento de novos aparelhos com multidetectores, é possível a realização mais rápida do exame tomográfico, além do uso de reformatações isométricas em todos os planos, possibilitando melhor avaliação de fraturas e lesões condrais relacionadas à instabilidade ${ }^{(1)}$.

\section{ULTRA-SOM}

O uso do ultra-som na instabilidade é limitado, utilizado usualmente para o diagnóstico diferencial de edema de partes moles, quando presente.

\section{CLASSIFICAÇÃO}

A classificação da clínica Mayo é a mais utilizada para as instabilidades do punho, classificando-as em instabilidades dissociativas e não-dissociativas ${ }^{(7)}$.
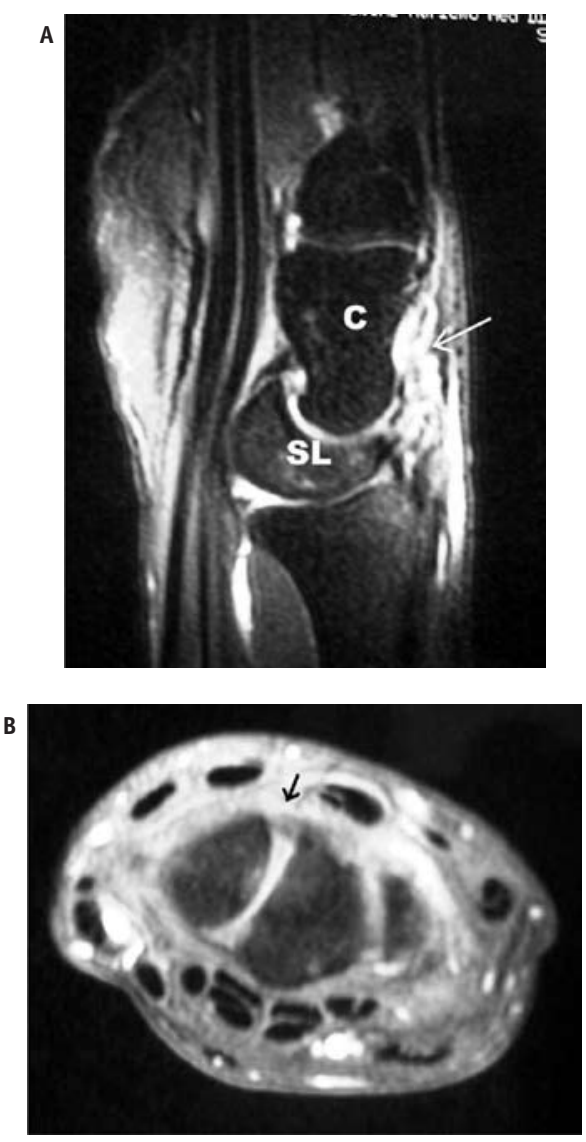

Figura 3 - Ressonância magnética do punho de paciente com instabilidade carpal em razão de lesões repetitivas. Cortes sagitais (a) e axiais (b) utilizando-se seqüência sensível a líquido, demonstrando edema/estiramento dos ligamentos extrínsecos dorsais (seta branca em a) e má definição por lesão da porção dorsal do ligamento escafosemilunar (seta preta em b).

$\mathrm{Na}$ instabilidade dissociativa, os elementos da fileira proximal estão desalinhados tanto no repouso quanto em movimento e estão relacionados à rotura dos ligamentos intrínsecos, mais comumente da fileira proximal ${ }^{(8)}$.

No grupo da instabilidade não-dissociativa, os elementos da fileira proximal estão intactos, porém há um desalinhamento em relação à fileira distal, com rotura ligamentar extrínseca $^{(8)}$. Há ainda um terceiro grupo, o complexo, que reúne elementos dos dois outros grupos. Neste artigo descreve-se o grupo de instabilidade dissociativa, com ênfase na DISI e VISI ${ }^{(1)}$.

\section{INSTABILIDADE DISSOCIATIVA}

Este grupo inclui as dissociações escafosemilunar e semilunarpiramidal. 


\section{DISSOCIAÇÃO ESCAFOSEMILUNAR}

Este é um dos padrões de instabilidade mais importante e pode estar associado com fratura do rádio e escafóide. Há várias formas de apresentação e estas foram divididas em quatro estágios, segundo Watson et al.

No estágio I há rotura parcial do LES, e as radiografias podem ser normais. No estágio II, o LES está completamente roto e as radiografias simples também podem estar normais. No estudo dinâmico, realizado quando há forte suspeita pelo exame clínico, podem-se observar aumento da distância entre o escafóide e o semilunar, e subluxação dorsal do escafóide ${ }^{(1)}$.

No estágio III, há rotura total do LES, porém com lesão nos estabilizadores secundários. Com a lesão da fixação ligamentar, o escafóide e o semilunar tendem a mover-se independentemente e de acordo com a tendência natural. $\mathrm{O}$ escafóide apresenta-se subluxado marcadamente em flexão, e o semilunar roda dorsalmente. O semilunar desloca-se até uma posição médio-volar, promovendo a subluxação do capitato. Essa deformidade é também chamada de instabilidade dorsofletida do segmento intercalado (DISI). Nas radiografias simples, o ângulo escafosemilunar está maior que $60^{\circ}$, e o ângulo semilunarcapitato maior que $30^{\circ}$ (Figura 4 ). Nas radiografias de frente, o semilunar apresenta-se com forma triangular, e o espaço escafosemilunar está aumentado, maior que $3 \mathrm{~mm}$ ( sinal do Terry-Thomas). O colapso em flexão do escafóide é caracterizado nas radiografias anteroposteriores como o sinal do anel, em razão da forma do pólo distal do escafóide ${ }^{(1,3)}$.

O estágio IV é considerado quando há alterações degenerativas na radiografia, acometendo as articulações radiocárpicas e mediocárpicas, ocorrendo ainda a interposição do capitato na articulação escafosemilunar (Figura 5$)^{(1)}$.
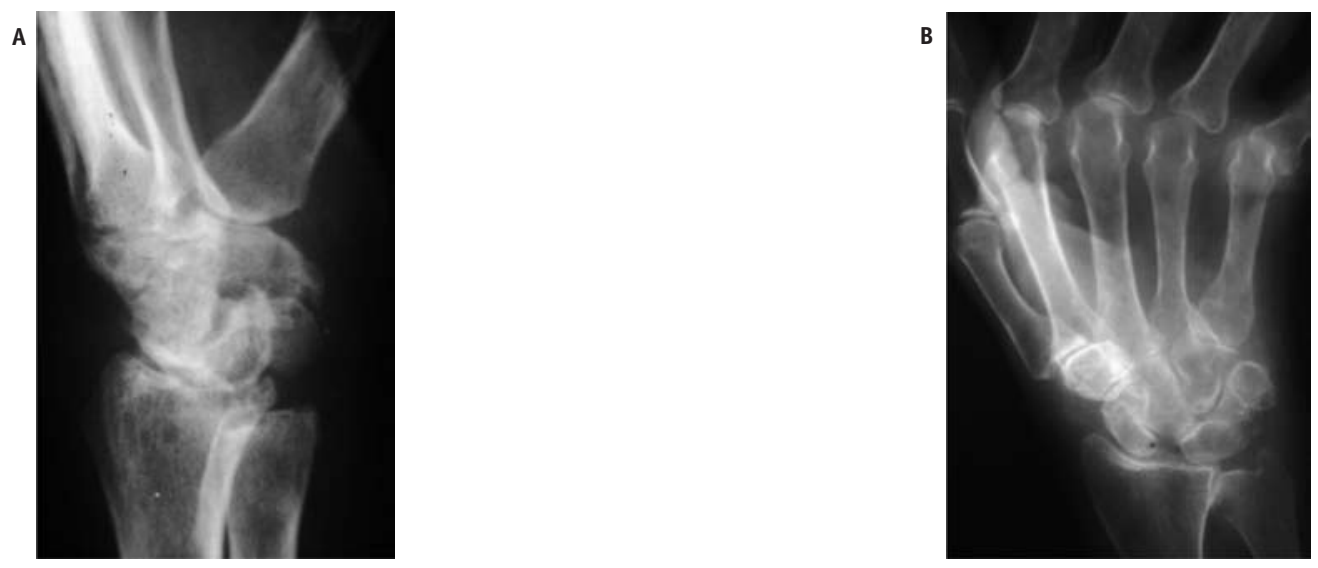

Figura 4 - DISI em paciente com artrite reumatóide. Radiografia em perfil (a), demonstrando aumento do ângulo escafosemilunar. Radiografia PA (b), evidenciando aumento da distância entre o escafóide e o semilunar (sinal do Terry Thomas) associado à leve migração proximal do capitato.
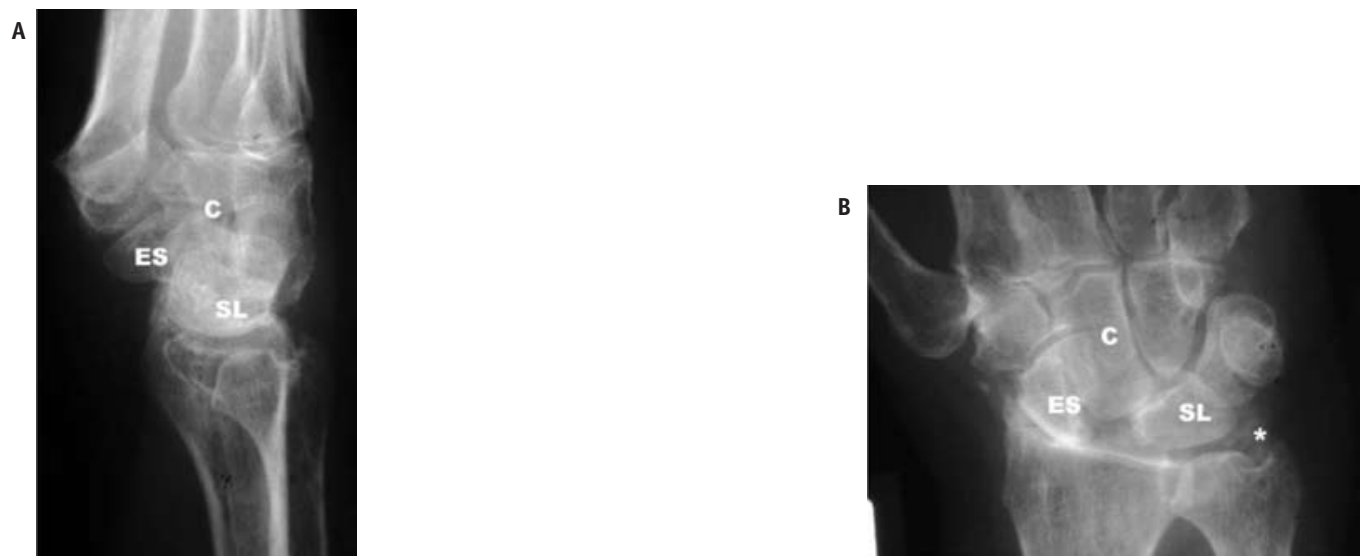

Figura 5 - Radiografias de perfil (a) e PA (b) de paciente com doença microcristalina (pirofosfato de cálcio), demonstrando DISI, com aumento dos ângulos semilunarcapitato e escafosemilunar, além do sinal de Terry Thomas e migração proximal do capitato. Há sinais de artropatia degenerativa radiocárpica e de calcificação da cartilagem triangular (asterisco). Escafóide (ES), semilunar (SL) e capitato (C). 


\section{DISSOCIAÇÃO SEMILUNARPIRAMIDAL}

Dissociação semilunarpiramidal (DSP) é rara e normalmente está associada à rotura não só do ligamento semilunar piramidal como dos ligamentos extrínsecos. Há aumento da flexão do semilunar que acompanha a tendência natural do escafóide, uma vez que o LES está intacto. Esta deformidade também é chamada de instabilidade volar do segmento intercalado (VISI).

É possível classificar a DSP em quatro estágios. No estágio I há lesão parcial e as radiografias são normais. No estágio II ocorre uma lesão completa do LSP, mas os ligamentos extrínsecos estão intactos; as radiografias simples não demonstram alterações, mas nas radiografias dinâmicas pode-se observar uma flexão do semilunar e do escafóide( ${ }^{(1)}$.

No estágio III, nas radiografias convencionais, há a flexão volar do escafóide e semilunar caracterizada em perfil, e nas radiografias de frente, o semilunar fica com forma de lua. $\mathrm{O}$ ângulo escafosemilunar fica reduzido (menor que $30^{\circ}$ ) e o ângulo semilunarcapitato, aumentado (maior que $30^{\circ}$ ). No estágio IV, sinais de osteoartrose são evidentes nas articulações cárpicas ${ }^{(1,3)}$.

\section{REFERÊNCIAS}

1. Schmitt R, Froehner S, Coblenz G, Christopoulos G: Carpal instability. Eur Rdiol 16: 2161-78, 2006.

2. Kauer JMG: The mechanism of carpal joint. Clin Orthop 202: 16-26, 1986.

3. Metz VM, Mtez-Schimmerl SM, Yin Y. Ligamentous instabilities of the wrist. European Journal of Radiology 25: 104-11, 1997.

4. Manuel J, Moran SL: The diagnosis and treatment of scapholunate instability. Orthop Clin Am 38: 261-77, 2007.

\section{CONCLUSÃO}

O diagnóstico da instabilidade carpal é um desafio para clínicos e radiologistas e deve ser feito com base em dados clínicos e estudos de imagem. Nos estágios tardios, seu diagnóstico é evidente, porém nos estágios iniciais as radiografias convencionais podem ser normais.

A avaliação inicial deve constar de radiografias convencionais, traçando-se os ângulos na radiografia de perfil e os arcos de Gilula na radiografia de frente. Se a avaliação inicial demonstrar alterações discretas, mas a clínica for sugestiva, devem ser realizados estudos dinâmicos.

Embora pouco usada para esse diagnóstico, a RM pode demonstrar a anatomia ligamentar e de partes moles, além de fornecer imagens de achados adicionais, auxiliando na eventual programação cirúrgica.

A TC possui papel secundário, ideal para avaliação da cortical óssea e das superfícies articulares.

A instabilidade carpal é uma condição freqüentemente negligenciada. Uma interação entre clínicos e radiologistas é essencial, o que pode levar ao correto diagnóstico, evitando-se estágios tardios, relacionados à alta morbidade, preservando a qualidade de vida dos pacientes.

5. Timins ME, Jahnke JP: MR imaging of the major carpal stabilizing ligaments: normal anatomy and clinical examples. Radiographics 15: 575-87, 1995.

6. Gilula LA: Carpal injuries: analytic approach and cases exercises. AJR 133: 503-17, 1979.

7. Amadio PC: Carpal kinematics and instability: a clinical and anatomic primer. Clin Anat 4: 1-12, 2005.

8. Lichtman DM, Wroten ES: Understanding midcarpal instability. The Journal of Hand Surgery 31: 491-8, 2006. 